\title{
Factores asociados a la invasión de pajas en bosques de la región semiárida central argentina
}

\author{
Ruth Rauber ${ }^{\varpi, 1,2}$; Diego SteinaKeR ${ }^{1,3} ;$ Manuel Demaría $^{1}$ \& Daniel Arroyo ${ }^{1}$ \\ 1. Instituto Nacional de Tecnología Agropecuaria, Villa Mercedes, Argentina. 2. CONICET. 3. University of Regina, \\ Department of Biology, Canada.
}

\begin{abstract}
Resumen. En los bosques de caldén (Prosopis caldenia) de la región semiárida central argentina, las pajas nativas no palatables C3 (Jarava ichu y Nassella tenuissima) han expandido su rango de distribución y abundancia, disminuyendo la diversidad vegetal y la productividad forrajera. Es posible que esta expansión esté relacionada al uso ganadero. Por lo tanto, resulta relevante evaluar la relación entre la intensidad de pastoreo, la abundancia de pajas y sus posibles efectos sobre factores edáficos. Se realizaron censos de vegetación y análisis de muestras de suelo a distinta intensidad de pastoreo y se encontró mayor abundancia de pajas en condiciones de pastoreo intermedio, relacionada positivamente con la cobertura de mantillo y negativamente con la materia orgánica del suelo y con la cobertura de suelo desnudo. El intenso disturbio y la competencia con otras especies podrían explicar la disminución de cobertura de pajas en uno y otro extremo del gradiente de pastoreo. El pastoreo afectó la composición de grupos funcionales a lo largo del gradiente, pero no la cobertura vegetal, la diversidad ni la riqueza de especies, la calidad nutricional del suelo, la cobertura de mantillo ni de suelo desnudo. La estabilidad de las funciones ecosistémicas podría deberse al reemplazo de especies que ocurre a través del gradiente que mantiene relativamente constante la cobertura vegetal total, siendo las especies ruderales las dominantes en los sitios extremadamente pastoreados, las pajas en los sitos con pastoreo intermedio, y las palatables junto a las pajas y latifoliadas en los sitios con pastoreo bajo.
\end{abstract}

[Palabras clave: pastoreo, recursos del suelo, palatabilidad, estrategias de uso de recursos]

\begin{abstract}
Aвstract. Factors associated to invasion by non-palatable grasses in Argentinean semi-arid forest: In semiarid Prosopis caldenia forests of the central region of Argentina, unpalatable native tussock grasses, Jarava $i c h u$ and Nassella tenuissima, have expanded their range and abundance, reducing plant diversity and forage productivity. This expansion may be related to domestic grazing. It becomes relevant, therefore, to evaluate the relationship between the intensity of grazing, the abundance of the tussock grasses, and its possible effects on soil factors. We made vegetation censuses and soil sample analyses in different grazing intensities and we found higher tussock grasses abundance at intermediate grazing intensity, positively related to litter cover, and negatively to soil organic matter and to bare soil cover. Intense disturbance, and competition with other species could explain its decrease at both grazing extremes. Grazing affected the functional group composition along gradient, but not the total plant cover, species diversity, richness, soil nutritional quality, litter cover, nor bare soil cover. The stability of the ecosystem functions may be supported by the species replacement taking place along the grazing gradient, which keeps constant the total plant cover: the ruderal species dominate heavily grazed sites, the tussock grasses dominate sites under moderate grazing, and a mix of ruderals, tussock grasses and palatable species occupy low grazed sites.
\end{abstract}

[Keywords: grazing, soil resources, palatability, resource use strategies]

\section{INTRODUCCIÓN}

Las invasiones biológicas constituyen una amenaza mundial para los ecosistemas (Mack et al. 2000; Kolar \& Lodge 2001). Las causas por las que una especie vegetal, nativa o exótica, invade un sistema pueden ser principalmente climáticas (Kulmatiski \& Beard 2013), genéticas (Mack et al. 2000), o antrópicas (Bellingham \& Coomes 2003). La contribución del hombre ha sido y sigue siendo muy importante en los eventos de invasiones biológicas, ya que, entre otras cosas, ha permitido la ruptura de las barreras geográficas que limitan la dispersión de la mayoría de las especies y ha alterado las comunidades nativas, principalmente

rauber.ruth@inta.gob.ar mediante sus actividades agrícola-ganaderas (Mack et al. 2000).

En sistemas ganaderos, algunas especies vegetales pueden resultar afectadas directamente por el comportamiento alimenticio selectivo de los herbívoros, por el disturbio provocado por el pisoteo, por el transporte de semillas en el pelaje y el sistema digestivo de los animales; e indirectamente por cambios en la intensidad y frecuencia de fuegos (Fleischner 1994; Vavra et al. 2007; Rogstad et al. 2009), y en la disponibilidad de agua y nutrientes en el suelo (Golluscio et al. 2009). En muchos casos, las especies beneficiadas aumentan su abundancia con

Recibido: 22 de octubre de 2013; Fin de arbitraje: 26 de diciembre; Última versión revisada: 2 de mayo; Aceptado: 13 de julio. 
respecto a las demás (MacLeod \& McIvor 2006) y pueden alcanzar coberturas tan altas que causan pérdida y fragmentación de hábitats (Milton \& Dean 1995), disminución de la oferta forrajera $y$, por lo tanto, de la producción ganadera (Milton \& Dean 1995; Eviner et al. 2010).

En los bosques de caldén (Cabrera 1976) de la región semiárida central argentina, las especies gramíneas nativas no palatables $\mathrm{C}_{3}$ Jarava ichu Ruiz \& Pav. (antes Stipa eriostachya Kunth) y Nassella tenuissima (Trin.) Barkworth (antes Stipa tenuissima Trin.), conocidas como "pajas", han expandido su rango de distribución y su abundancia (Llorens 1995; Busso 1997; Menéndez \& La Rocca 2006; Morici et al. 2009). La superficie boscosas que aún se conserva se utiliza principalmente con fines ganaderos (Morici et al. 2009) y cuando las pajas dominan el estrato herbáceo de estos ecosistemas, disminuye la diversidad vegetal $\mathrm{y}$, debido a su baja palatabilidad, también la productividad forrajera (Menéndez \& La Rocca 2006; Morici et al. 2009).

La expansión de pajas en estos ecosistemas podría ser resultado del aumento en la presión de pastoreo bovino, un fenómeno generalizado en la región semiárida central argentina como consecuencia del corrimiento de la frontera agrícola-ganadera y redistribución de gran parte del stock bovino desde regiones subhúmedas-húmedas a regiones semiáridas (INTA-Senasa 2009). Este aumento de la presión ganadera favorecería a las especies no palatables, directamente por selectividad animal, e indirectamente por reducción del nitrógeno disponible para las plantas (Golluscio et al. 2009). Moretto y Distel (1997) propusieron para el Caldenal un modelo conceptual que plantea que la preferencia animal aumenta la proporción de las especies menos preferidas por el ganado y a su vez, disminuye la concentración de nitrógeno en el suelo. Esto se debería a que las especies menos preferidas tienen menor concentración de nitrógeno y mayor de fibra, y ofrecen un mantillo con menor tasa de descomposición que las más preferidas. Como consecuencia, en áreas intensamente pastoreadas el suelo se empobrecería progresivamente en nitrógeno originandounmecanismoderetroalimentación positivo que beneficiaría a las especies menos palatables, más tolerantes a estas condiciones de baja disponibilidad de nutrientes (Moretto \& Distel 1997). La baja palatabilidad de las pajas es una característica típica de especies tolerantes a estrés (Grime 1977).
A pesar de las observaciones parciales realizadas con respecto a la invasión de pajas en la región semiárida central argentina, no se han realizado estudios que evalúen la relación entre la abundancia de pajas, la intensidad de pastoreo y calidad nutricional del suelo. El objetivo general de este estudio fue evaluar esta relación. Para esto se analizó si: a) la abundancia de pajas es mayor en condiciones de mayor intensidad de pastoreo, b) se observa un deterioro en la vegetación, esto es, disminución de la riqueza y la diversidad y aumento de la cobertura de suelo desnudo a medida que aumenta la intensidad de pastoreo y c) la calidad nutricional del suelo (materia orgánica, nitrógeno, fósforo) es menor en sitio con alta abundancia de pajas. Se espera que la cobertura de pajas aumente y la riqueza, la diversidad y la calidad del suelo disminuya con la intensidad del pastoreo.

\section{Materiales y MÉTOdOS}

\section{Área de estudio}

El estudio se llevó a cabo en bosques de caldén (Prosopis caldenia Burkart) ubicados al sureste de la provincia de San Luis, Argentina, entre los $34^{\circ} 51^{\prime} \mathrm{S}$, $65^{\circ} 06^{\prime} \mathrm{O}$ y $\operatorname{los} 35^{\circ} 30^{\prime} \mathrm{S}, 6^{\circ} 55^{\prime} \mathrm{O}$. El Distrito del Caldén, perteneciente a la Provincia Fitogeográfica del Espinal (Cabrera 1976), se extiende en el centro y sureste de la provincia de San Luis, ocupando originalmente unas 600.000 ha al suroeste de la provincia de Córdoba, centro de la provincia de La Pampa y sur de Buenos Aires (Anderson et al. 1970). Esta región posee un gradiente climático con precipitaciones medias anuales crecientes en dirección noreste-suroeste (de 450 a $650 \mathrm{~mm}$ ), con una temperatura media de $15,3^{\circ} \mathrm{C}$ (Adema et al. 2003; Fernández et al. 2009). La comunidad vegetal clímax estaría constituida por un bosque abierto de caldén con árboles de 8 a $10 \mathrm{~m}$ de altura, con un estrato arbustivo pobre y un estrato herbáceo principalmente compuesto de gramíneas $\mathrm{C}_{4}$ (Cabrera 1976; Gallego \& Distel 2004). Además presenta dos condiciones de luminosidad dadas por la densidad del dosel arbóreo; un sotobosque con presencia de especies umbrófilas y el abra, donde se encuentran especies heliófilas (Orquín et al., 1983). Los suelos dominantes corresponden a los órdenes entisoles (ustortente típico y ustipsamente típico) y molisoles (haplustol éntico y fluvéntico). El régimen hídrico es ústico, que en general indica humedad restringida, aunque en determinado momento permite el crecimiento normal de las plantas (Peña Zubiate et al. 1998). La textura del suelo varía entre franca y franco arenosa, y el $\mathrm{pH}$ entre 6 y 8 . Sus características más importantes son la semiaridez del clima, la costra calcárea (tosca), el escaso contenido de materia orgánica (1.5-3\%), el drenaje algo excesivo y la susceptibilidad a los procesos erosivos (Adema et al. 2003; Martín et al. 2008). 


\section{Relevamiento de campo}

Se utilizaron gradientes de pastoreo dados por distancias a la aguada (Nazar Anchorena 1988). En base a imágenes satelitales y recorridos a campo se seleccionaron siete potreros de más de 400 ha con una sola aguada, en los que la estimación visual de cobertura de pajas era mayor al 30\%. En cada uno de estos siete potreros se ubicó la aguada, a partir de la cual se estableció una transecta de $2500 \mathrm{~m}$ de largo, a $45^{\circ}$ de los alambrados o en la dirección que permitiera llegar a la distancia máxima, en el caso de potreros rectangulares. A lo largo de la transecta, se seleccionaron tres puntos a diferentes distancias de la aguada: $200 \mathrm{~m}, 1000 \mathrm{~m}$ y 2500 $\mathrm{m}$, que corresponden a distintas condiciones de presión de pastoreo, pastoreo alto, medio y bajo, respectivamente (Nazar Anchorena 1988). En cada una de estas distancias se registró la abundancia (\% de cobertura) de todas las especies vegetales, suelo desnudo y mantillo, sobre 3 transectas de $30 \mathrm{~m}$ de largo, perpendiculares a la primera, mediante el método de intersección de puntos (Herrick et al. 2005) y tomando un registro cada metro. Todos los datos se tomaron en 15 días. Las transectas se establecieron separadas por más de $10 \mathrm{~m}$ entre sí debido a que las especies gramíneas de la zona presentan una correlación espacial a distancias menores (Echeverría \& Giulietti 1991). El ganado presente fue siempre vacuno, con cargas que varían entre 5 y 8 ha por equivalente vaca.

Adicionalmente, se tomaron muestras de suelo (0-20 cm de profundidad), a los $0 \mathrm{~m}, 15 \mathrm{~m}$ y $30 \mathrm{~m}$ de cada transecta. Estas muestras se mezclaron y homogeneizaron, obteniendo una sola para cada transecta, que fueron sometidas a análisis químico

Tabla 1. Valores medios y error estándar de variables edáficas y de vegetación bajo distinta intensidad de pastoreo.

Table 1. Mean values and standard error of edaphic and vegetation variables under different grazing intensities.

\begin{tabular}{lccc}
\hline \multirow{2}{*}{ Variable } & \multicolumn{3}{c}{ Intensidad de pastoreo } \\
\cline { 2 - 4 } $\begin{array}{l}\text { Materia orgánica } \\
\text { (\% gr) }\end{array}$ & $1.21 \pm 0.18$ & $1.12 \pm 0.11$ & $1.26 \pm 0.12$ \\
Nitratos (ppm) & $7.05 \pm 0.92$ & $6.24 \pm 0.67$ & $6.29 \pm 0.72$ \\
Amonio (mg/kg) & $4.14 \pm 0.49$ & $4.14 \pm 0.30$ & $4.33 \pm 0.80$ \\
Nitrógeno total & $0.74 \pm 0.07$ & $0.89 \pm 0.12$ & $0.77 \pm 0.06$ \\
(mg/g) & & & \\
Fósforo (ppm) & $30.67 \pm 3.12$ & $33.9 \pm 2.55$ & $33.24 \pm 3.22$ \\
C/N & $0.71 \pm 0.06$ & $0.67 \pm 0.05$ & $0.74 \pm 0.05$ \\
Conductividad & $0.46 \pm 0.07$ & $0.40 \pm 0.08$ & $0.38 \pm 0.03$ \\
(mS/cm) & & & \\
pH & $6.18 \pm 0.14$ & $6.01 \pm 0.12$ & $6.11 \pm 0.12$ \\
Riqueza & $8.95 \pm 0.58$ & $8.76 \pm 0.40$ & $9.71 \pm 0.57$ \\
Diversidad (S-W) & $1.8 \pm 0.07$ & $1.81 \pm 0.06$ & $1.92 \pm 0.07$ \\
Cobertura vegetal & $137.8 \pm 8.3$ & $154.0 \pm 7.8$ & $141.3 \pm 8.8$ \\
total (\%) & & & \\
Suelo desnudo (\%) & $25.1 \pm 4.2$ & $21.0 \pm 4.7$ & $30.7 \pm 3.2$ \\
Mantillo (\%) & $56.0 \pm 7.2$ & $65.8 \pm 5.1$ & $56.0 \pm 4.9$ \\
\hline
\end{tabular}

para determinar el contenido de materia orgánica y carbono, nitrógeno total, nitrato y amonio (SNEDD, Marbán \& Ratto 2005), el pH (relación suelo:agua 1: $2,5)$ y la conductividad eléctrica (en pasta).

\section{Análisis estadísticos}

Para evaluar si a) la abundancia de pajas es mayor y b) se observa deterioro de la vegetación en condiciones de mayor intensidad de pastoreo se realizaron análisis de varianza. Se evaluó diferencias de abundancia de pajas, todas las especies palatables, todas las especies no palatables, todas las latifoliadas, especies C3 y C4, cobertura vegetal total, riqueza, diversidad (Shannon- Weaver) suelo desnudo, mantillo a lo largo del gradiente de pastoreo. Para el caso de las variables que presentaron distribución normal, se compararon los tratamientos mediante un test LSD Fisher. Si la distribución no resultó normal, se realizó un test de Kruskal-Wallis. Para estos análisis se utilizó el programa InfoStat v. 2012.

Para estudiar si la calidad nutricional del suelo es menor en sitio con alta abundancia de pajas y para evaluar la relación de las variables edáficas con las especies presentes se realizó un Análisis de Correspondencia Canónico. Para esto se tuvieron en cuenta solo las especies presentes en más de 5 transectas, y las variables edáficas no correlacionadas entre sí que presentaron mayor asociación a los ejes de ordenamiento. Para este análisis se utilizó el programa PCOrd v 5.0.

\section{Resultados}

Las especies registradas se clasificaron en diferentes categorías (ver Información Suplementaria). Se observó mayor riqueza de especies no palatables con respecto a las palatables en el área de estudio. La abundancia de las pajas fue mayor en condiciones de intensidad de pastoreo media (Figura 1), al igual que el conjunto de especies C3. Cuando se consideraron todas las especies no palatables (Información suplementaria), la abundancia fue mayor en condiciones de pastoreo medio con respecto a intensidad baja (Figura 1). La abundancia de cobertura de especies palatables fue mayor en intensidad baja de pastoreo, mientras que la abundancia de latifoliadas fue mayor en intensidad alta, aunque no hubo diferencias dentro de los grupos de especies C4, suelo desnudo ni mantillo (Figura 1). No hubo variación significativa a lo largo del gradiente de pastoreo en la riqueza, la diversidad, la cobertura vegetal total, la cobertura de suelo desnudo y de mantillo (Tabla 1). Ninguna de las variables de suelo analizadas presentó diferencias estadísticamente significativas a lo largo del gradiente estudiado (Tabla 1). 


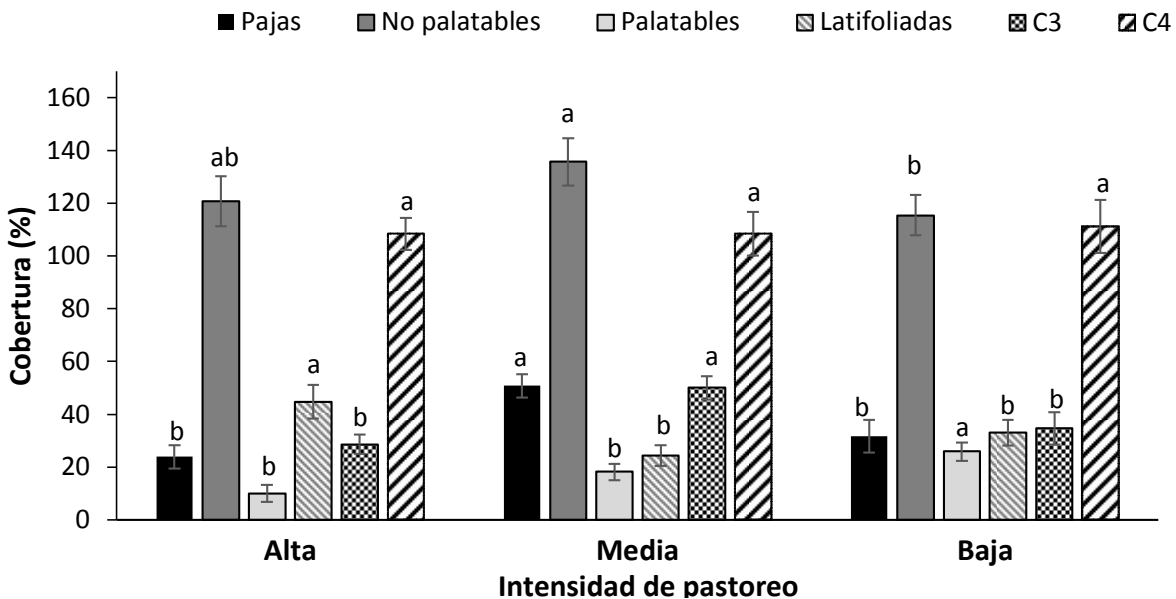

Figura 1. Abundancia media ( $\mathrm{N}=7$ ) de cada tipo vegetal, suelo desnudo y mantillo, para tres intensidades de pastoreo. Los valores son porcentajes de cobertura aérea absoluta. Dentro de la categoría de especies no palatables se encuentran incluidas las gramíneas no palatables, las pajas, las latifoliadas y leñosas. En las categorías C3 y C4 se agruparon las especies de acuerdo a su ruta metabólica, independientemente de su palatabilidad. Prueba Kruskal-Wallis, $\mathrm{p}<0.05$ para evaluar diferencias de cobertura media de pajas. Las demás variables fueron analizadas con la prueba LSD Fisher, $\mathrm{p}<0.05$. Letras diferentes indican diferencias significativas de cobertura de cada tipo funcional entre intensidades de pastoreo.

Figure 1. Mean abundance $(\mathrm{N}=7)$ of each plant type, bare soil and litter for three grazing intensities. Values are percentages of total air cover. Within the category of unpalatable species are included woody unpalatable grasses, straw, and hardwoods.

El análisis multivariado muestra que las dos especies de pajas se encuentran asociadas positivamente a la abundancia de mantillo y a una intensidad media de pastoreo y negativamente con la cantidad de materia orgánica y con la cobertura de suelo desnudo (Figura 2). Por otra parte, no se observan agrupaciones claras de las demás especies individuales en cuanto a su palatabilidad, su ruta metabólica ni su forma de hoja (Figura 2). Las especies que más explican el eje I son C. montevidensis, B. ulicinia, P. caldenia, D. califórnica y $P$. lanuginosa, así como todas las variables ambientales estudiadas. El eje II está explicado principalmente por la especie $S$. kali y las variables ambientales (Tabla 2).

\section{DisCUSIÓN}

En este sistema el pastoreo afectó la composición de grupos funcionales pero no la cobertura vegetal, la diversidad ni la riqueza de especies. Tampoco afectó la calidad nutricional del suelo, la cobertura de

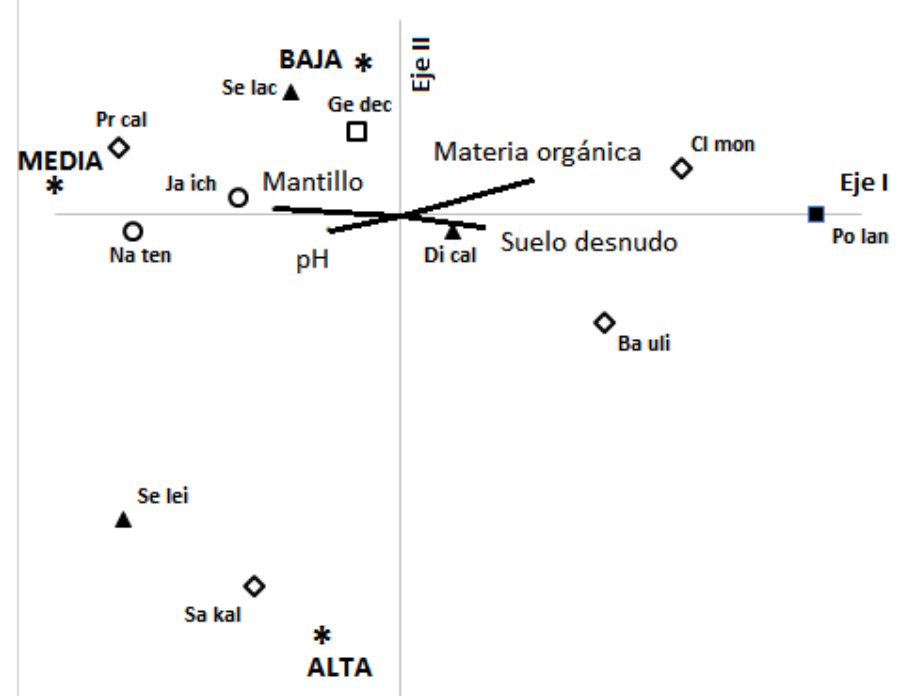

Figura 2. Análisis de Correspondencia Canónico. Inercia $=1.50$, Autovalor Eje $\mathrm{I}=0.346$, Eje II=0.052. Símbolos vacíos: no palatables, llenos: palatables, círculos: pajas, rombos: latifoliadas, cuadrados: C3, triángulos: C4. Los asteriscos representan la intensidad de pastoreo. Especies: $\mathrm{Ba}$ uli $=$ Baccaris ulicinia, $\mathrm{Cl}$ mon $=$ Clematis montevidensis, Di cal= Digitaria califórnica, Ge dec= Geoffroea decoricans, Ja ich= Jarava ichu, $\mathrm{Na}$ ten $=$ Nassella tennuissima, Po lan $=$ Poa lanuginosa, $\mathrm{Pr} \mathrm{cal}=$ Prosopis caldenia, $\mathrm{Sa}$ $\mathrm{kal}=$ Salsola $\mathrm{kali}, \mathrm{Se}$ lac $=$ Setaria lachnea, $\mathrm{Se}$ lei=Setaria leiantha. Los ejes I y II explican el $85.1 \%$ de la variabilidad total (63.3 y $21.8 \%$ respectivamente).

Figure 2. Canonical Correspondence Analysis. Inertia $=1.50$, Eigenvalue $=$ Axis $\mathrm{I}=0.346$, Axis II $=0.052$. Empty symbols: non-palatable, filled: palatable, circles: "pajas", rhombus: broadleaves, squares: C3, triangles: $\mathrm{C} 4$. Asterisks represent the grazing intensity. 
Tabla 2. Asociaciones de cada variable con cada uno de los ejes del Análisis de Correspondencia Canónico. ${ }^{*} \mathrm{p}<0.05$, ** $\mathrm{p}<0.01,{ }^{* * *} \mathrm{p}<0.001$. Para el análisis se tomaron las especies presentes en más de 5 transectas, y las variables edáficas no correlacionadas entre sí que presentaron mayor asociación a los ejes de ordenamiento.

Table 2. Association of each variable to Canonical Correspondence Analysis axes. ${ }^{*} \mathrm{p}<0.05,{ }^{* *} \mathrm{p}<0.01$, *** $\mathrm{p}<0.001$.

\begin{tabular}{lcc}
\hline & Eje I & Eje II \\
\hline Jarava ichu & $-0250^{*}$ & 0.026 \\
Nassella tennuissima & $-0.288^{*}$ & 0.075 \\
Prosopis caldenia & $-0.457^{* * *}$ & $0.284^{*}$ \\
Clematis montevidensis & $0.874^{* * *}$ & $0.223^{*}$ \\
Setaria lachnea & -0.027 & $0.271^{*}$ \\
Baccaris ulicinia & $0.720^{* * *}$ & -0.065 \\
Digitaria californica & $0.349^{* * *}$ & 0.097 \\
Setaria leiantha & $-0.234^{*}$ & $-0.237^{*}$ \\
Geoffroea decorticans & 0.065 & 0.150 \\
Poa lanuginosa & $0.571^{* * *}$ & 0.066 \\
Salsola kali & -0.059 & $-0.289^{* *}$ \\
Materia orgánica & $0.625^{* * *}$ & $0.568^{* * *}$ \\
Suelo desnudo & $0.528^{* * *}$ & $-0.581^{* * *}$ \\
Mantillo & $-0.913^{* * *}$ & $0.312^{* *}$ \\
pH & $-0.546^{* * *}$ & $-0.696^{* * *}$ \\
\hline
\end{tabular}

mantillo ni de suelo desnudo. Esto sugiere que a lo largo del gradiente de intensidad de pastoreo existiría un remplazo de grupos de especies que mantendrían las distintas funciones del ecosistema. Por su parte, la abundancia de pajas fue mayor a intensidad media de pastoreo, y no a intensidad alta, como esperábamos encontrar. Adicionalmente, las altas coberturas de pajas estuvieron relacionadas a una baja cantidad de materia orgánica del suelo, baja cobertura de suelo desnudo y alta cobertura de mantillo.

La abundancia de pajas aumentaría significativamente de baja a moderada intensidad de pastoreo hasta un máximo, y luego disminuiría con alta presión de pastoreo (Bestelmeyer 2006; Brieske et al. 2006). Sin embargo, el patrón de invasión observado no se corresponde con la disminución de la cobertura vegetal, a diferencia de lo que mostraron estudios previos, donde se observó una mayor abundancia de $N$. tenuissima en sitios con mayor cobertura de suelo desnudo que en sitios con abundante vegetación (Busso 1997).

Diversos estudios demuestran que el pastoreo altera las relaciones competitivas de las especies, favoreciendo a algunas en detrimento de otras (Parker et al. 2006; Díaz et al. 2007).
En condiciones de pastoreo intenso, las especies latifoliadas presentaron mayor cobertura y las especies palatables dominaron en intensidad de pastoreo baja. Esta distribución de los grupos funcionales a lo largo del gradiente de pastoreo parece responder a las estrategias dominantes de las poblaciones, siguiendo el modelo C-S-R propuesto por Grime (1977). Este modelo establece tres estrategias adaptativas en las plantas: competidoras, tolerantes al estrés y ruderales y sus estados intermedios. Varias especies de ambientes semiáridos poseen características que les confieren baja palatabilidad y alta tolerancia al estrés (Moreno et al. 2010) y, en el marco de este modelo, las pajas tolerarían eficientemente el estrés causado por el pastoreo moderado. En condiciones de baja presión de pastoreo, en cambio, las especies palatables podrían limitar la expansión de las pajas a través por competencia (Crawley 1990). En el otro extremo, en condiciones de mayor disturbio provocado por el sobrepastoreo, mayor pisoteo o incluso pastoreo sobre especies de baja palatabilidad, y por la mayor disponibilidad de nitrógeno (Mattson 1980; Díaz et al. 2007), las especies latifoliadas anuales de rápido crecimiento prevalecen y disminuye la abundancia de las pajas. Este resultado coincide con los encontrados en pastizales costeros de California, donde las latifoliadas exóticas anuales aumentaron bajo pastoreo doméstico (Skaer et al. 2013).

La abundancia de pajas estuvo relacionada negativamente con la cantidad de materia orgánica del suelo cuando se analizaron los datos independientemente de la intensidad de pastoreo y a una escala de análisis espacial menor, evaluando la relación de las variables sitio a sitio. Sin embargo, estas variables de suelo y otras indicadoras de calidad como amonio, nitrógeno total, $\mathrm{pH}$, suelo desnudo y mantillo, no cambiaron a lo largo de los gradientes de pastoreo evaluado. La baja cantidad de nitrógeno en los tejidos de las pajas explicaría la relación positiva entre la abundancia de pajas y cobertura de mantillo, y negativa con la cantidad de materia orgánica y cobertura de suelo desnudo. El mantillo de las pajas sería menos lábil, debido a su mayor relación $\mathrm{C} / \mathrm{N}$ en sus tejidos, lo que determinaría que en los sitios de mayor abundancia de pajas haya mayor cobertura de mantillo y menor materia orgánica, incluso al compararlo con los sitios sometidos a menor presión de pastoreo, donde se esperaría encontrar mayor cobertura de broza por menor 
pisoteo (Fleichner 1994). Esta relación negativa entre las pajas y la materia orgánica del suelo indicaría que existe un deterioro de la calidad nutricional del suelo a medida que aumenta la abundancia de las invasoras, o bien, que la abundancia de pajas es consecuencia del deterioro. En ambos casos, el sistema resultaría en una posible retroalimentación positiva en favor de las invasoras.

Numerosos estudios han documentado reducción de riqueza, abundancia, biomasa, cobertura y concentración de nutrientes en el suelo en relación al pastoreo (Reeder \& Schuman 2002; Singer \& Schoenecker 2003; Bilotta et al. 2007; Han et al. 2008; Tessema et al. 2011). En los bosques de caldén, en cambio, no se observan diferencias en la calidad nutricional del suelo, al igual que en otros sistemas ganaderos (Quiroga et al. 2009). La estabilidad de estas funciones ecosistémicas podría deberse al reemplazo de especies que ocurre en dicho gradiente y mantiene constante la cobertura vegetal total: las especies ruderales (principalmente latifoliadas anuales) dominaron sitios extremadamente pastoreados, las pajas en sitos con intermedia intensidad de pastoreo, y las palatables junto a las pajas y latifoliadas los sitios con pastoreo moderado a bajo.

El proceso que determina la invasión de pajas en ambientes del caldenal es sumamente complejo y aparte de los factores antrópicos, edáficos y poblacionales parcialmente analizados, otros factores ambientales como la abundancia de las precipitaciones podrían ejercer un rol preponderante. En los últimos años se registró un aumento de las precipitaciones con respecto a los registros históricos de la región. En condiciones de estrés hídrico, las especies $\mathrm{C}_{4}$ se encontrarían en ventaja competitiva frente a las $C_{3}$, debido a su mayor eficiencia en el uso del agua. Sin embargo, cuando las limitaciones hídricas disminuyen por aumento de las precipitaciones, esta ventaja competitiva disminuiría, debido a que la fijación de carbono en las especies $\mathrm{C}_{4}$ implica mayor gasto energético con respecto a la ruta de fijación de las especies $\mathrm{C}_{3}$.

Un aumento en las presiones de pastoreo junto al incremento de las precipitaciones podría favorecer la invasión de pajas en la región con una disminución de las capacidades de carga ganadera de los campos. Este estudio analiza algunas de las causas que favorecen la invasión de pajas en bosques de caldén, pero es necesario profundizar los análisis para brindar herramientas que garanticen un manejo ganadero adecuado que limite las invasiones de especies no deseables y permita la utilización sustentable de este ecosistema.

Agradecimientos: Agradecemos a los productores agropecuarios que nos permitieron realizar los relevamientos en sus campos y a $\mathrm{K}$ Frigerio por el asesoramiento estadístico. También agradecemos a los revisores anónimos y a la editora, cuyos comentarios ayudaron a mejorar el trabajo. Este trabajo se llevó a cabo mediante fondos de financiación del proyecto regional PAMSL-820082 del Instituto Nacional de Tecnología Agropecuaria y con beca posdoctoral de CONICET. Este trabajo cumple con las normas éticas vigentes para la República Argentina.

\section{BiBLIOGRAFÍA}

Adema, EO; FJ Babinec; DE Buschiazzo; MJ Martín \& N PeInEMANN. 2003. Erosión hídrica en los suelos del Caldenal. INTA, EEA Anguil. Publicación Técnica N 53.

Anderson, DL; JA Del Aguila, \& AE Bernardón. 1970. Las Formaciones Vegetales en la Provincia de San Luis. RIA. S.2. 7:153-183.

Bellingham, PJ \& DA Coomes. 2003. Grazing and community structure as determinants of invasion success by Scotch broom in a New Zealand montane shrubland. Div. Distr., 9:19-28.

Bestelmeyer, BT. 2006. Threshold Concepts and Their Use in Rangeland Management and Restoration: The Good, the Bad, and the Insidious. Rest. Ecol., 14:325- 329.

Bilotta, GS; Brazier, RE \& PM Haygarth. 2007. The impacts of grazing animals on the quality of soils, vegetation, and surface waters in intensively managed grasslands. Advances in Agronomy 94:237-250.

BRISKE, DD; SD FuHLENDORF \& FE SMEINS. 2006. A unified framework for assessment and application of ecological thresholds. Rang. Ecol. Manag., 59:225-236.

Busso, CA. 1997. Towards an increased and sustainable production in semi-arid rangelands of central Argentina: two decades of research. J. Arid Env., 36:197-210.

Cabrera, AL. 1976. Regiones Fitogeográficas Argentinas. En: Tomo, Fascículo II (Ed.), Enciclopedia Argentina de Agricultura y Ganadería, 2a.edición. Acme S.A.C.I, Buenos Aires, Argentina.

Coley, PD; JP Bryant \& FS ChapIN III. 1985. Resource availability and plant anti-herbivore defense. Science, 230:895-899.

CRAWLEY, MJ. 1990. Rabbit grazing, plant competition and seedling recruitment in acid grassland. J. Appl. Ecol., 27: 803-820.

Diaz, S; S Lavorel; S Mcintyre; V FalczuK, F CASANOVES; ET AL. 2007. Plant trait responses to grazing - a global synthesis. Global Change Biology ,13:313-341.

ECHEVERRíA, JC \& JD GiUlietTI. 1991. Variabilidad especial de las gramíneas en un pastizal natural de San Luis. Revista Argentina de Producción Animal, 11:29-34.

EVINER, VT; SA Hoskinson \& CV HAWKEs. 2010. Ecosystem impacts of exotic plants can feed back to increase invasion in western US rangelands. Rangelands, 31:21-31.

FERNANDEZ, OA; ME GIL \& RA Distel. 2009. The challenge of rangeland degradation in a temperate semiarid region of Argentina: The caldenal. Land degradation and development, 20:431-440. 
FLeISCHNER, TL. 1994. Ecological costs of livestock grazing in Western North America. Conserv. Biol., 8:629- 644.

Gallego, L \& RA Distel. 2004. Phytolith Assemblages in Grasses Native to Central Argentina. Ann. Bot., 94: 865-874.

Golluscio, RA; AT Austin; GC Garcia Martinez; M.Gonzalez-Polo; OE Sala \& RB Jackson. 2009. Sheep grazing decreases organic carbon and nitrogen pools in the Patagonian steppe: Combination of direct and indirect effects. Ecosystems, 12:686-697.

GRIME, JP. 1977. Evidence for the existence of three primary strategies in plants and its relevance to ecological and evolutionary theory. Am. Nat., 111:169-1194.

Han, G; X HaO; M ZhaO; M WanG; BH EllerT; W WillmS \& M WANG. 2008. Effect of grazing intensity on carbon and nitrogen in soil and vegetation in a meadow steppe in Inner Mongolia. Agr. Ecosyst. Env., 125:21-32.

Herrick, JE; JW Van Zee; KM Havstad; LM Burkett \& WG WHITFORD. 2005. Monitoring manual for grassland, shrubland and savanna ecosystems. USDA-ARS Jornada Experimental Range. The University of Arizona Press, Tucson, Arizona, USA, p. 236.

InTA-SENASA. 2009. Stock 2009 del ganado bovino de carne. Información técnica $\mathrm{N}^{\circ}$ 174. Ediciones INTA. Buenos Aires, Argentina.

INTA. Buenos Aires, Argentina. Kolar, C \& D Lodge. 2001. Progress in invasion biology: predicting invaders. Trends Ecol. Evol., 16:199- 204.

Kulmatiski, A \& KH Beard. 2013. Woody plant encroachment facilitated by increased precipitation intensity. Nature Climate Change, doi: 10.1038/ nclimate1904.

LLORENS, EM. 1995. Viewpoint: The state and transition model applied to the herbaceous layer of Argentina's calden forest. J. Range Manage. 48: 442- 447.

Mack, R; D Simberloff; WM Lonsdale; H Evans; M Clout \& F Bazzaz. 2000. Invasiones Biológicas: Causas, Epidemiología, Consecuencias globales y Control. Tópicos en Ecología, 5:1-19.

Macleod, N \& J Mcivor. 2006. Reconciling economic and ecological con icts for sustained management of grazing lands. Ecol. Econ. 56, International Society for Ecological Economics, Maryland, pp. 386-401.

Marbán, L \& S RAtTo. 2005. Tecnologías en análisis de suelo. Grancharoff Impresores. Buenos Aires.

Martín, J; E Adema; S Aimar \& F Babinec. 2008. Efecto del rolado sobre propiedades fisicoquímicas del suelo en el ecotono Caldenal-Monte Occidental. INTA EEA Anguil. Publicación técnica $\mathrm{N}^{\circ} 76$.

Mattson, WJ Jr. 1980. Herbivory in Relation to Plant Nitrogen Content. Ann. Rev. Ecol. Syst., 11: 119-161.

MenÉndez, JL \& SM La Rocca. 2006. Primer inventario nacional de bosques nativos. Informe Regional Espinal, Segunda Etapa. ANEXO I: Estado de Conservación del Distrito Caldén. Fundación Bosques de la Patagonia. http:/ / www.drn.lapampa.gov.ar/BosquesyPastizales / FloraNativa/Estado_de_Conservacion_del_Distrito_ Calden.pdf.
Milton, SJ \& WRJ Dean. 1995. South Africa's Arid and Semiarid Rangelands: Why are they changing and can they be restored?. Environmental Monitoring and Assessment, 37: 245-64.

Moreno, L; MB Bertiller \& A Carrera. 2010. Changes in traits of shrub canopies across an aridity gradient in northern Patagonia, Argentina. Basic Appl. Ecol., 11: 693-701.

MoRETto, AS \& RA Distel. 1997. Competitive interactions between palatable and unpalatable grasses native to a temperate semi-arid grassland of Argentina. Plant Ecol., 130: 155-161.

Morici E; V Doménech-GarCía; G GÓMEZ-CASTRO; A Kin; A SAENZ \& C RABOTNIKOF. 2009. Diferencias estructurales entre parches de pastizal del Caldenal y su influencia sobre el banco de semillas, en la provincia de La Pampa, Argentina. Agrociencia, 43:529-537.

Nazar Anchorena, JB. 1988. Pastizales naturales de La Pampa. Manejo de los mismos. AACREA-Prov. de La Pampa. $112 \mathrm{p}$.

Orquín, L; D Losada; M Delgado; E Gabutti \& J Bertón. 1983. El estado de degradación de la vegetación en un área del bosque de caldén (Prosopis caldenia Burk.) IDIA Sup. 36:224-230.

Parker, JD; DE Burkepile \& ME Hay. 2006. Opposing effects of native and exotic herbivores on plant invasions. Science, 311:1459-1461.

Peña Zubiate, CA; DL Anderson; MA Demmi; JL Saenz \& A D’Hiriart. 1998. Carta de suelos y vegetación de la Provincia de San Luis. Secretaría de Agric., Ganadería, Pesca y Alimentación; INTA y Gobierno de la Prov. de San Luis. Est. Exp. Agr. San Luis. 115 pp.

Quiroga, A; Fernández R \& E Noellemeyer. 2009. Grazing effect on soil properties in conventional and no-till systems. Soil Till. Res., 105:164-170.

ReEDer, JD \& GE Schuman. 2002. Influence of livestock grazing on $C$ sequestration in semi-arid mixed-grass and short-grass rangelands. Environmental Pollution, 116:457-463.

Rogstad, A; TM Bean; A Olsson \& GM CASADy. 2009. Fire and invasive species management in hot deserts: resources, strategies, tactics, and response. Rangelands, 31:6-13.

Singers, FJ \& KA SchoenecKer. 2003. Do ungulates accelerate or decelerate nitrogen cycling? Forest Ecol Manag, 181:189-204.

SKaer, MJ; DJ Graydon \& JH Cushman. 2013. Communitylevel consequences of cattle grazing for an invaded grassland: variable responses of native and exotic vegetation. J. Veg. Sc., 24:332-343.

Tessema, ZK; WF De Boer; RMT BaArs \& HHT Prins. 2011. Changes in soil nutrients, vegetation structure and herbaceous biomass in response to grazing in a semi-arid savanna of Ethiopia. J. Arid Env., 75:662-670.

VAVRA, M; CG PARKS \& MJ WISDOM. 2007. Biodiversity, exotic plant species, and herbivory: the good, the bad, and the ungulate. For. Ecol. Manage., 246:66-72 


\section{INFORMACIÓN SUPLEMENTARIA}

Especies vegetales registradas. Fuentes bibliográficas para nombrar las especies: Zuloaga y Morrone 1996, 1999, Zuloaga et al. 1994, actualizadas en http://www2.darwin.edu.ar/ Proyectos/FloraArgentina/FA.asp y en http:/ /www.floraargentina.edu.ar/

Registered plant species. Bibliographic sources to species names: Zuloaga and Morrone 1996, 1999,Zuloaga et al. 1994, actualized in http:/ / www2.darwin.edu.ar/Proyectos/FloraArgentina/ FA.asp y en http:/ / www.floraargentina.edu.ar/

\begin{tabular}{|c|c|}
\hline Palatables & No palatables \\
\hline gramíneas perennes $\mathrm{C} 4$ & gramíneas perennes $\mathrm{C} 4$ \\
\hline Digitaria californica (Benth.) Henrard & Pappophorum pappiferum (Lam.) Kuntze \\
\hline Sporobolus cryptandrus (Torr.) A. Gray & Cenchrus pauciflorus Benth \\
\hline Setaria leucopila (Scribn. \& Merr.) K. Schum & gramíneas perennes $\mathrm{C} 3$ \\
\hline Setaria lachnea (Nees) Kunth & Jarava ichu Ruiz \& Pav \\
\hline Eustachys retusa (Lag.) Kunth & Nasella tenuissima (Trin.) Barkworth \\
\hline Melica argyrea Hack & Amelichloa brachychaeta (Godr.) Arriaga \& Barkworth \\
\hline Aristida inversa Hack & latifoliadas \\
\hline A. mendocina Phil & Clematis montevidensis Spreng \\
\hline Botriochloa springfieldii (Gould) Parodi & Baccharis ulicina Hook. \& Arn \\
\hline Cynodon hirsutus Stent & Heterotheca subaxillaris (Lam.) Britton \& Rusby \\
\hline gramíneas perennes $\mathrm{C} 3$ & Bidens subalternans DC \\
\hline Piptochaetium napostanense (Speg.) Hack & Gaillardia megapotamica (Spreng.) Baker \\
\hline Poa ligularis Nees ex Steud & Salsola kali $\mathrm{L}$ \\
\hline Poa lanuginosa Poir & Cyperus sp \\
\hline gramínea anual & Baccharis gilliesii A. Gray \\
\hline Bromus brevis Steud & Aloysia grattissima (Gill. et Hook) Tronc \\
\hline arbustiva & Lippia turbinata Griseb \\
\hline \multirow[t]{18}{*}{ Lycium chilense Miers } & leñosas \\
\hline & Maytenus angustifolia Mattos \& N.F. Mattos \\
\hline & Jodina rhombifolia (Hook. \& Arn.) Reissek \\
\hline & Schinus fasciculata (Griseb.) I.M. Johnst \\
\hline & Eupatorium patens D. Don ex Hook. \& Arn \\
\hline & Prosopis chilensis (Molina) Stuntz emend. Burkart \\
\hline & Geoffroea decorticans (Gillies ex Hook. \& Arn.) Burkart \\
\hline & Condalia microphylla Cav. \\
\hline & Prosopis alpataco Phil \\
\hline & Capsicum chacoënse Hunz \\
\hline & Cestrum parqui L'Hér \\
\hline & Prosopis flexuosa DC \\
\hline & Chenopodium album $\mathrm{L}$ \\
\hline & Baccharis crispa Spreng \\
\hline & Gomphrena sp \\
\hline & Lycium sp \\
\hline & Larrea divaricata Cav \\
\hline & Senna aphylla (Cav.) H.S. Irwin \& Barneby \\
\hline
\end{tabular}

